\title{
Cuestiones en torno a la filosofía de la India. Tendencias académicas en las universidades de Argentina y dilemas (meta)filosóficos.
}

\author{
Gabriel Martino ${ }^{1}$
}

UBA, CONICET, UCA

Artículo científico. Material original autorizado para su primera publicación en Journal de Ciencias Sociales, Revista Académica de la Facultad de Ciencias Sociales de la Universidad de Palermo.

Recibido: 8-8-2015

Aceptado: 2-10-2015

\section{Resumen}

En el presente trabajo nos proponemos abordar diferentes cuestiones relativas a la filosofía de la India. Examinamos, en primer término, la situación actual del área en las universidades argentinas y, específicamente, en el currículo de las licenciaturas en filosofía dictadas en nuestro país. Relevamos, con esta finalidad, el programa de estudios de las treinta y dos carreras de grado en filosofía ofrecidas por las universidades públicas y privadas nacionales. En segundo término, brindamos una concisa discusión de la bibliografía especializada y actualizada respecto del dilema acerca de la existencia o no de una filosofía de la India. En relación con esta discusión, abordamos algunos ejemplos de autores que procuran desarticular aquellas posiciones arraigadas y centenarias que sostienen que no hay filosofía en India. Ofrecemos, asimismo, la visión propuesta por los especialistas contemporáneos en el pensamiento indio que defiende la presencia de la filosofía en la cultura de la India clásica y que argumenta a partir de un conocimiento sistemático y metodológicamente honesto acerca de esta tradición. Nuestra investigación procura poner de manifiesto, que el abordaje del dilema (meta)filosófico acerca de si hay o no filosofía en la India requiere e implica una tradición de investigación (auto)crítica especializada en el área y que en nuestro país tal tradición se encuentra en un estado embrionario.

Palabras clave: filosofía de la India, orientalismo, racionalidad, plan de estudio de filosofía en Argentina

\footnotetext{
${ }^{1}$ Licenciado y Doctor en Filosofía (UBA). Becario posdoctoral CONICET. Profesor de Textos de Filosofía Antigua, Facultad de Filosofía y Letras de la Universidad Católica Argentina y Adscripto a la Cátedra de Sánscrito de la Facultad de Filosofía y Letras, UBA. Mail: gabriel.filosofia@hotmail.com
} 


\section{Abstract}

In the present paper we discuss different issues concerning the Philosophy of India. We examine, in the first place, the current situation of the area in Argentinean universities and, more specifically, in the programme of the Licenciaturas in Philosophy taught in our country. We assess, with this purpose, the programme of the thirty two degrees in Philosophy offered by national private and public universities. In the second place, we provide a brief discussion of the up-to-date specialized bibliography about the dilemma concerning the existence or not of philosophy in India. We consider, thus, the possitions of a few authors who try to deconstruct centenary and rooted conceptions about the absence of philosophy in India. We evaluate, as well, the stances of contemporary specialists in Indian thought who defend the presence of philosophy in classical Indian culture arguing from a sistematic and methodologically honest knowledge of this tradition. Our inquiry tries to show, in such a way, that approaching the (meta)philosophical dilemma regarding the presence or absence of philosophy in India requires and implies a (self-)critical and specialized tradition of research in the area, and that in our country this tradition exists only in an very incipient stage.

Keywords: Indian Philosophy, orientalism, rationality, study programmes in Philosophy in Argentina

\section{Introducción}

En el año 2008 los investigadores argentinos ${ }^{2}$ Carmen Dragonetti y Fernando Tola publicaron el volumen Filosofía de la India. El mito de la oposición entre "pensamiento" indio y "filosofía" occidental, en el cual sostienen cuatro tesis cuyo núcleo común consiste en indicar la consonancia temática y metodológica entre la filosofía griega y europea, por un lado, y el pensamiento Indio, por otro. ${ }^{3}$ En esta obra, mediante un minucioso análisis comparativo de un vasto número de fuentes filosóficas occidentales y de textos sánscritos Tola y Dragonetti defienden, tal como el título mismo del volumen pone de manifiesto, que "sí existió «tal cosa como una Filosofía de la India»". ${ }^{4}$

Así pues, una vez que el lector se adentra en la disciplina gracias a este volumen especializado encuentra que, junto con nombres como Platón, Aristóteles, Tomás de Aquino, Leibniz, Spinoza y Kant, se mencionan

\footnotetext{
${ }^{2}$ La Dra. Carmen Dragonetti es argentina mientras que el Dr. Tola es peruano de nacimiento. Ambos, sin embargo, se encuentran radicados en Argentina desde la década del 70. Ambos han trabajado en el CONICET como Investigadores superiores y dirigen hace veinticinco años la Fundación Instituto de Estudios Budistas radicada en la Ciudad de Buenos Aires. Asimismo, el libro Filosofía de la India citado, tal como sus autores afirman en la página 17, fue escrito con el apoyo de la Agencia Nacional de Promoción Científica y Tecnológica de nuestro país que les concedió dos subsidios para desarrollar dos proyectos de investigación cuyas conclusiones se presentan en ese volumen.

${ }^{3}$ Tola - Dragonetti (2008). Allí los autores defienden con amplio respaldo textual las cuatro tesis siguientes: (1) Por lo menos hasta el siglo XVII India, por un lado, y Grecia y Europa, por otro, reflexionan muchas veces sobre los mismos temas filosóficos y de la misma manera. (2) En la historia de las filosofías griega y europea se encuentran manifestaciones de irracionalidad bajo muchas formas y son tan numerosas como en la historia del pensamiento de la India. (3) En la India sí existió tal cosa como una Filosofía de la India. (4) La comparación entre el pensamiento indio y el occidental debe limitarse a confrontar a ambos tal como se manifestaron antes del siglo XVII o incluso en los siglos siguientes pero, en este caso, cuando ellos mantienen formas que prolongan las filosofías previas a esa fecha.

${ }^{4}$ Tola - Dragonetti (2008, p. 23). La cursiva es de los autores.
} 
otros como Bādarāyaṇa, Śan்kara, Rāmānuja, Madhva, Bhartṛhari, entre tantos otros. Aprende, asimismo, que paralelamente al platonismo, al aristotelismo y al estoicismo en Grecia, por ejemplo, en la India antigua se desarrollaron corrientes filosóficas clásicas llamadas Sānikhya, Yoga y Vedānta, entre otras. Y lee también que las concepciones idealistas o materialistas, teístas o ateas occidentales, por ejemplo, pueden ser contrastadas con formulaciones análogas de concepciones idealistas o materialistas, teístas o ateas indias. Todo este recorrido, guiado por la pluma de dos investigadores que escriben en nuestra propia lengua, lo lleva a la siguiente conclusión tras setecientas páginas de examen sistemático: “Como consecuencia de las anteriores observaciones, es posible afirmar que sí existió Filosofía en India como en Occidente, con las mismas expectativas, con las mismas debilidades $-\mathrm{y}$ queda así eliminado el mito de Filosofía sólo en Occidente y sólo Pensamiento en la India." ${ }^{5}$

Ahora bien, dado que, de acuerdo con el punto de vista calificado de dos especialistas locales de renombre internacional, la filosofía parece ser algo propio tanto de los occidentales como de los indios, es esperable que algún curso formal de instrucción en filosofía dictado en nuestro país incluya en su currículo no solo la discusión y la enseñanza de las concepciones de los filósofos de Occidente sino también la de los filósofos indios, al menos la de los más influyentes. Vale la pena examinar, pues, si de las instituciones universitarias públicas y privadas argentinas donde se dicta una Licenciatura en Filosofía, alguna contiene en su programa de estudios una orientación o módulo o materia dedicada a la filosofía de la India. En la próxima sección analizamos, entonces, la situación de tales estudios en los establecimientos de nuestro país. En una sección subsiguiente, a su vez, examinamos con cierto detalle la polémica desarrollada en las últimas décadas en torno a la (in)existencia de la filosofía de la India, en la cual la voz de los autores Tola y Dragonetti ya citados se encuentra incluida. Estas dos secciones procuran sustentar, en fin, nuestra hipótesis según la cual la indagación en torno al carácter de la producción intelectual India y su relación con la filosofía implica ineludiblemente una tradición de investigación (auto)crítica especializada en el área, sin la cual toda representación del pensamiento indio es insustancial y caricaturesca.

\section{Tendencias académicas en las universidades de Argentina}

En nuestro país hay treinta centros educativos superiores que otorgan el título oficial de Licenciado en Filosofía, y dos de ellos poseen, además, la posibilidad de obtener el título bajo la modalidad "a distancia". ${ }^{6}$

\footnotetext{
${ }^{5}$ Tola - Dragonetti (2008, p. 702).

${ }^{6}$ Las treinta y dos Licenciaturas en filosofía relevadas en nuestra investigación son las dictadas en las siguientes instituciones del país: Facultad de Filosofía, Ciencias de la Educación y Humanidades, Universidad de Morón; Humanidades, Universidad Nacional Santo Tomás de Aquino; Facultad de Humanidades, Ciencias Sociales y de la Salud, Universidad Nacional Santiago del Estero; Humanidades, Universidad Católica de La Plata; Facultad de Filosofía, Ciencias de la Educación y Humanidades, Universidad Católica de Santa Fe; Facultad de Filosofía y Letras, Universidad del Salvador; Facultad de Filosofía y Letras, Universidad de Buenos
} 
De las treinta y dos Licenciaturas en Filosofía totales cuyo plan de estudios vigente hemos podido relevar, sin embargo, ninguna de ellas incluye la materia Filosofía de la India. Tres de ellas, no obstante, poseen una materia en la cual se incluyen elementos de la filosofía de la India. Describamos con cierto detalle estos tres casos.

La Licenciatura en Filosofía dictada por la Facultad de Educación y Humanidades de la Universidad de Morón, por una parte, incluye en su tercer año la materia Filosofía de Oriente, de duración cuatrimestral y con una carga horaria de seis horas semanales. La asignatura está a cargo del Lic. Ezequiel Ramoneda, egresado de la Licenciatura en Estudios Orientales de la Universidad del Salvador, y tiene como objetivo, tal como se afirma en su programa, "introducir al estudiante en las principales tradiciones filosóficas clásicas de los pueblos Indio y Chino". El cronograma está estructurado en cuatro unidades en las cuales se abordan, primero, a modo de introducción, las diferencias entre las filosofías occidental y oriental y entre la india y la china. Se dedica una segunda unidad, "Filosofía India", al estudio de las diferentes corrientes de pensamiento de la India, tanto ortodoxas ("Nyaya y Vaisesika", "Samkhya y Yoga", "Purva Mimamsa y Uttara Mimamsa") como heterodoxas ("Lokayata. Materialismo" y "Jainismo"). La tercera unidad "Filosofía China" está dedicada al análisis de diversas escuelas y textos propios del pensamiento chino y, por último, la unidad "Filosofía Budista" aborda las diferentes tradiciones filosóficas del budismo ("Theravada (Hinayana)", "Mahayana", "Vijñanavada" y "Sunyavada"). Cabe destacar que las tres unidades dedicadas a las tradiciones india, china y budista incluyen como primer elemento su contextualización histórica y caracterización general, lo cual favorece, a nuestro entender, la comprensión de los contenidos abordados desde una perspectiva situada que evita, a su vez, las lecturas anacrónicas y esencialistas. ${ }^{7}$

Por otra parte, la Licenciatura ofrecida por la Facultad de Humanidades, Ciencias Sociales y de la Salud de la Universidad Nacional de Santiago del Estero, contiene en su plan la materia Pensamiento de Oriente incluida en el primer año curricular, de duración cuatrimestral y con una carga horaria de cuatro horas semanales. La asignatura está a cargo del doctor en filosofía (UCSF) Alejandro Auat, y desde el corriente año también de la licenciada Susana Alonso, y se aborda con la "intención de superar el eurocentrismo y occidentalismo de la visión normalizada de la historia de la filosofía." Vale la pena señalar que en este caso la aproximación a las

\footnotetext{
Aires; Facultad de Humanidades y Ciencias de la Educación, Universidad Nacional de la Plata; Facultad de Filosofía y Letras, Universidad Católica Argentina; Facultad de Humanidades Y Ciencias de la Educación, Universidad de Mar del Plata; Departamento de Humanidades, Universidad Nacional del Sur; Centro de Estudios de la Orden de Predicadores, Universidad Nacional Santo Tomás de Aquino; Facultad de Humanidades y Artes, Universidad Nacional de Rosario; Facultad de Humanidades, Universidad Nacional de Salta; Facultad de Filosofía, Universidad Nacional de San Juan; Facultad de Humanidades y Ciencia, Universidad Nacional del Litoral; Facultad de Humanidades, Universidad Nacional de Catamarca; Facultad de Humanidades, Universidad Nacional del Nordeste; Facultad de Ciencias Humanas, Universidad Nacional de Río Cuarto; Facultad de Filosofía y Letras, Universidad Nacional de Tucumán; Facultad de Filosofía y Humanidades, Universidad Nacional de Córdoba; Facultad de Humanidades, Universidad Nacional del Comahue; Escuela de Humanidades, Universidad Nacional de General de San Martín; Facultad de Artes y Ciencias, Universidad Católica de Salta; Psicología y Ciencias Sociales, Universidad de Ciencias Empresariales y Sociales; Facultad De Humanidades, Artes Y Ciencias Sociales, Universidad Autónoma de Entre Ríos; Facultad de Humanidades y Ciencias Sociales, Universidad Nacional de Jujuy; Facultad de Filosofía y Humanidades, Universidad Católica de Córdoba; Facultad de Filosofía y Letras, Universidad Nacional de Cuyo; Universidad Nacional de Tres de Febrero. Las dos instituciones relevadas que dictan la licenciatura en filosofía en la modalidad "a distancia" son la Facultad de Humanidades de la Universidad Católica de la Plata y el programa Untrefvirtual de la Universidad de Tres de Febrero.

7 Agradecemos al Lic. Ramoneda por habernos facilitado el programa de la asignatura a su cargo.
} 
expresiones filosóficas de Oriente se realiza desde un marco teórico que el programa explicita como "la hipótesis del tiempo-eje de Karl Jaspers, que nos brinda un punto de apoyo para la comprensión universal de la historia del pensamiento. Hipótesis que será matizada desde la visión de los sistemas inter-regionales de las altas culturas." La primera unidad del programa, "EL TIEMPO-EJE", está dedicada al estudio del marco teórico antes mencionado. Las siguientes dos unidades están destinadas a "LA FILOSOFÍA CHINA" (U. 2) y a "LAS FILOSOFÍAS DE LA INDIA" (U. 3). Destaquemos de la última unidad que se encaran tres sistemas diferentes: el Budismo, el Brahmanismo y el Jainismo. Esto pone de manifiesto, a nuestro entender, que se busca abarcar en la medida de lo posible las corrientes ortodoxas y heterodoxas respecto de la compleja construcción llamada hinduismo sin limitarse a la tradición brahmánica. ${ }^{8}$

La licenciatura en Filosofía dictada por el Departamento de Humanidades de la Universidad Nacional del Sur (Bahía Blanca, Prov. de Buenos Aires), a su vez, contiene la materia Historia de la Filosofía Oriental. La asignatura está incluida en el cuarto año de la carrera, posee una duración cuatrimestral y una carga horaria de seis horas semanales distribuidas en clases teóricas (4 horas) y en clases prácticas (2 horas). Los profesores a cargo son los licenciados Guillermo Goicochea y Karen Garrote, ambos egresados de la carrera de Filosofía de la UNS. El programa está estructurado en cuatro unidades. La primera, "Oriente-Occidente", encara la definición de "Oriente", la metodología y los problemas propios de la disciplina a la que la asignatura corresponde, así como dos diferentes concepciones de “Orientalismo" (Said, Sardar). La segunda unidad "India", incluye el estudio del Vedismo, del Brahmanismo, del Hinduismo y del Budismo. La tercera, está dedicada el estudio de China y la cuarta de Japón, tradición, esta última, sobre la que los profesores, a nuestro entender, han profundizado con mayor detalle en sus estudios. Vale la pena resaltar que, también en este caso, el programa de la materia refleja y problematiza el marco teórico-metodológico desde el cual se abordan los contenidos. En sus "Objetivos", en efecto, se explicita con claridad que se busca "Ingresar a la problemática que abre el enfrentarse a un pensar que no se reclama de nuestra tradición, tratando de acercar nuestro horizonte de comprensión, sin caer en la reducción y manipulación del pensamiento oriental como un mero objeto cargado de exotismo". ${ }^{9}$

Los tres casos mencionados, pues, agotan las posibilidades que los estudiantes de las Licenciaturas en Filosofía de las universidades argentinas, tanto públicas como privadas, tanto laicas como religiosas, poseen de adentrarse formalmente en el marco de sus estudios universitarios de grado a la(s) filosofía(s) de la India. Como nuestro relevo pone de manifiesto, dos de las Universidades en las que se incluyen las materias (la Universidad Nacional del Sur y la Universidad Nacional de Santiago del Estero) son públicas, mientras que

\footnotetext{
${ }^{8}$ Agradecemos al Dr. Auat por habernos facilitado los programas de la materia a su cargo, tanto el vigente hasta el año pasado como la nueva versión vigente a partir del segundo cuatrimestre del corriente año.

${ }^{9}$ Agradecemos a Yamil Balercia del departamento de Humanidades de la UNS por habernos facilitado el programa de la materia.
} 
una de ellas, la Universidad de Morón, es privada. Llama la atención que ninguna otra universidad privada del país incluya en su programa de estudio de la carrera de filosofía, en muchos casos de reciente creación, la materia de filosofía de la India o de Oriente. Es cierto, por otra parte, que los especialistas en el tema son escasos y ciertamente la gran mayoría de las universidades no cuentan con una tradición de formación de estudiosos en el área que puedan encarar el dictado de esta clase de materias, por lo cual dependerían, en este sentido, de la acotada disponibilidad que las (tres únicas) universidades mencionadas tienen de proveerles de especialistas. Permítasenos aclarar que no creemos que el hecho de diplomarse en una carrera que dispone de una sola materia en el área implique que el egresado sea un especialista en ella. Consideramos, sin embargo, que la existencia de tal asignatura habilita no sólo el espacio de la cursada sino también otras modalidades de pertenencia y de actividad que favorecen la investigación y la especialización en el área. Pensemos, por ejemplo, que un alumno podría realizar su tesis de licenciatura en uno de los temas abordados en la cursada de la asignatura aludida bajo la dirección del docente. Esto le otorgaría al licenciado un primer perfilamiento en el área y lo habilitaría al cursado de estudios de mayor especialidad en la misma línea. ${ }^{10}$

Examinemos, por último, un cuarto caso que si bien no corresponde a una Licenciatura en filosofía merece, a nuestro entender, ser tomado en cuenta. La Universidad del Salvador posee como una de sus dependencias la Escuela de Estudios Orientales, centro fundado por el Padre Ismael Quiles en 1967, originalmente dependiente de la Facultad de Filosofía, de la cual se separa en 1976 y se constituye como Escuela dependiente del Rectorado. ${ }^{11}$ Desde entonces en la Escuela se dicta la Licenciatura en Estudios Orientales, carrera de cinco años de duración, que en su tercer año incluye la materia Religiones y filosofías de la India. ${ }^{12}$ La materia tiene una duración cuatrimestral y una carga horaria de tres horas por semana, está a cargo de los licenciados. Liliana García Daris y Carlos González Laporte, ambos egresados de la Licenciatura en Estudios Orientales. El programa, debido su especificidad, aborda una cantidad considerable de temáticas propias del área como "Las Enseñanzas Upanishadicas" (U. 1), "Muerte e inmortalidad" (U. 2), “Karma y trasmigración" (U. 3), "El Brahman" (U. 4), "La Trimurti. Brahma, Vishnu y Shiva” (U. 5), Los sistemas ortodoxos (U. 6) y los heterodoxos (U. 7). En relación con este cuarto caso creemos que vale la pena señalar, en primer lugar, que el Lic. Ezequiel Ramoneda, profesor de la Universidad de Morón para la materia Filosofía de Oriente es egresado de esta Escuela. Este hecho pone de manifiesto, a nuestro entender, un interesante fenómeno de complementación entre las especialidades e, incluso, entre las

\footnotetext{
${ }^{10}$ La inexistencia de una universidad en nuestro país que ofrezca un doctorado en filosofía de la India profundiza, no obstante, el problema que se ve, a su vez, reforzado por la reducida cantidad de especialistas argentinos que podrían dirigir un doctorado en este área.

11 http://eeo.usal.edu.ar/ consultada en Julio de 2015.

12 Años más tarde se incluye el dictado de la Tecnicatura Universitaria de Yoga y de la Tecnicatura en China Contemporánea. La primera de ellas incluye dos materias cuatrimestrales también dedicadas a la filosofía de la India.
} 
instituciones, ya que si bien el Prof. Ramoneda no es licenciado en filosofía, su especialidad en el área de estudios orientales lo facultaría al dictado de la asignatura en una licenciatura en filosofía. Este intercambio, sin embargo, lamentablemente es aislado. Resulta interesante notar, en este sentido, que la Licenciatura en Filosofía de la Universidad del Salvador carece hasta el momento, al menos, de una asignatura dedicada al estudio de la filosofía de la India o de Oriente, algo que llama la atención dado que la Universidad cuenta con una Escuela de la que egresan especialistas en estudios orientales que, en un caso por lo menos, dictan una materia semejante en una universidad pública. Adviértase, por último, que la orientación y el contenido de la asignatura dictada en la Escuela no son netamente filosóficos ya que, tal como su nombre lo indica y como el programa parece poner de manifiesto, no se establece una distinción nítida entre el fenómeno cultural de corte filosófico del de corte religioso. Así pues, si bien se trata de la única licenciatura del país en la que se aborda el estudio de la filosofía específicamente de la India, tal estudio se encuentra amalgamado con el de las religiones de la India. Tal amalgama, aparentemente, más que indicar una inclusión conjunta de temáticas diferentes parece poner de manifiesto una cierta asimilación de ambos fenómenos en la cual sus bordes no se distinguen. Ahora bien, tratándose de una carrera orientada al estudio de fenómenos culturales amplios que no se acotan a lo meramente filosófico, el abordaje de la asignatura resulta, a nuestro entender, adecuado y coherente con el marco propio de la Licenciatura en la que se incluye. ${ }^{13}$

Tal es, pues, de acuerdo con el relevo que hemos llevado a cabo, el panorama nacional del estudio formal universitario a nivel de grado de la filosofía de la India. Se trata, a nuestro entender, de un panorama que evidencia un momento embrionario del desarrollo de estos estudios en las universidades del país. Mas tal carácter incipiente no solo es puesto de manifiesto por la acotada proporción de instituciones cuyas carreras de grado en filosofía incluyen el abordaje de la temática (menor al diez por ciento), ni por el reducido espacio dentro del currículo del que dispone, ${ }^{14}$ ni por el reducido número de alumnos que accede a tales estudios. ${ }^{15}$ Consideremos, por ejemplo, que las universidades públicas de los grandes centros urbanos como Córdoba, Rosario, La Plata y la Ciudad Autónoma de Buenos Aires, ${ }^{16}$ de las cuales egresan la mayor cantidad de licenciados en filosofía del país, no imparten estos conocimientos. Observemos,

\footnotetext{
${ }^{13}$ Agradecemos al Prof. Lic. Carlos Rúa por habernos recibido en la Escuela y por habernos facilitado los programas de las materias Filosofías y religiones de la India y los diferentes programas de la materia Introducción a la Filosofía de Tradición India de la Tecnicatura Universitaria de Yoga.

${ }^{14}$ La carga horaria de las materias dedicadas a la filosofía oriental constituye alrededor de un 3-4\% aproximadamente de la carga horaria total de las Licenciaturas en las que se incluyen.

${ }^{15}$ No hemos logrado calcular el porcentaje que surge de contrastar la cantidad de alumnos que cursan las materias aludidas en las tres licenciaturas mencionadas con la totalidad de alumnos de las licenciaturas en filosofía de todo el país. Pero debido a la distribución desigual de estudiantes en las diferentes universidades y a su concentración mayoritaria en universidades que carecen de esta materia, podemos estimar que si estas tres licenciaturas constituyen un $9,4 \%$ del total de licenciaturas en filosofía, los alumnos que asisten a ellas seguramente constituyen una proporción considerablemente menor a esta.

${ }^{16}$ No podemos dejar de mencionar que la Licenciatura en Letras Clásicas de la Facultad de Filosofía y Letras de la Universidad de Buenos Aires incluye la única Cátedra de Sánscrito del país, que todos los años dicta un curso anual. La materia está dividida en clases teóricas a cargo de la Dra. Rosalía Vofchuk, con una carga de cuatro horas semanales, y clases prácticas a cargo de la Dra. Gabiela Müller, de dos horas por semana. El programa de la materia incluye la exposición de algunos elementos propios de la filosofía india y el trabajo de traducción de textos filosóficos sánscritos. En el marco de esta cátedra realicé, luego de haber cursado y aprobado la materia, una adscripción de cuatro años que me permitió incluir como parte de mi investigación doctoral temáticas propias de la filosofía de la India. Actualmente continúo mis investigaciones posdoctorales en torno a la filosofía de la India y a la filosofía de la tardo-antigüedad griega desde una perspectiva comparativa y bajo la co-dirección de Rosalía Vofchuk, profesora a cargo de la Cátedra.
} 
asimismo, la falta de interconexión entre los centros y los especialistas que se dedican al área, la ausencia de asociaciones o grupos que los nuclee ${ }^{17}$ y la inexistencia de eventos de difusión de alcance nacional dedicados a la filosofía de la India o, incluso de "Oriente". Tomemos en cuenta, también, que las materias en las que se incluyen los contenidos relativos a la filosofía de la India están destinadas, en los tres primeros casos, a la filosofía de "Oriente". Tal denominación implica, a nuestro entender, variadas cuestiones que vale la pena analizar.

Poseer una materia cuatrimestral dedicada a la filosofía de "Oriente", en primer término, reduce los contenidos relativos a la filosofía de la India a una unidad en una asignatura. Englobar las filosofías de las tradiciones india, china y japonesa, por lo menos, bajo el rótulo de "orientales" da, asimismo, la dudosa imagen de una cuestionable homogeneidad o de un problemático parentesco entre estas tres tradiciones y de su conjunta alteridad respecto de la filosofía "occidental". Tal modo de aproximarse a las tradiciones corre el riesgo de caer en una concepción esencialista que las imagina como entidades monolíticas, impermeables e inconmensurables: Oriente por un lado, Occidente, por otro. ${ }^{18}$ Respecto de este último punto, creemos que a partir de nuestro examen de los tres planes de estudio que contienen esta materia, complementado con el de los veintinueve planes que no incluyen ninguna asignatura relativa a filosofías "no occidentales", podríamos afirmar que tales concepciones problemáticas se ven plasmadas en la estructuración del currículo de las licenciaturas y que la aproximación (o exclusión) orientalista a los estudios de la India (y de las otras tradiciones "orientales") subyace en ellas. Diferente es el panorama, sin embargo, delineado por los tres programas específicos de las materias en los que se estudia la filosofía de "Oriente", donde se evidencia no solo un conocimiento de la problemática del orientalismo sino que también se la tematiza, se la cuestiona, y se proponen marcos teóricos alternativos para el estudio de las tradiciones mencionadas. Esta disonancia entre el plan de estudios de las licenciaturas en filosofía y los programas de las materias específicas aludidas es indicador, según creemos, de que en el reducido núcleo del estudio y enseñanza universitaria de la filosofía de la India (y de las otras tradiciones) los docentes se encuentran, respecto de su especialidad, un paso adelante del currículo correspondiente al cual incorporan su aporte. Este índice, a nuestro entender, es favorable y es el requisito indispensable para que, eventualmente, los filósofos implicados puedan ejercer su influencia produciendo la reconfiguración de concepciones de la filosofía en retroceso y dar cabida a nuevos modos de concebir la disciplina y sus

\footnotetext{
17 Mencionemos al menos a la rama Argentina de la Asociación Latinoamericana de estudios de Asia y África (ALADAA) que nuclea, no obstante, a profesionales de diversas disciplinas que exceden ampliamente, aunque se la incluye, a la filosofía de la India.

${ }^{18}$ Acerca de la noción de inconmensurabilidad en el abordaje intercultural de la filosofía véase Barua (2013: 5-32).
} 
alcances, modos que en el ámbito internacional parecen haber florecido hace ya unas cuantas décadas. ${ }^{19}$ En aquellas universidades del país en las cuales no hay siquiera tal disonancia, el panorama parecería ser más estático y menos propenso al cambio y a la actualización, al menos respecto del asunto que discutimos en el presente artículo.

\section{Dilemas (meta) filosóficos}

La cuestión de si es posible afirmar o no la existencia de una (o varias) filosofía(s) de la India, sin embargo, es compleja, se ha discutido incluso por varios siglos y nuestra comprensión de esta problemática no debe reducirse a un análisis de las tendencias propias de las instituciones nacionales o internacionales. En la presente sección, pues, examinamos el dilema aludido y discutimos las posiciones de algunos referentes internacionales en el tema de modo de alcanzar una comprensión mayor del problema.

Comencemos refiriéndonos a un pasaje que podemos leer en uno de los textos más utilizados a nivel mundial en las últimas cuatro décadas para el estudio de la filosofía griega clásica, área ineludible para todo abordaje académico de la filosofía: "Los motivos y los métodos de las escuelas indias y el trasfondo teológico y místico de su pensamiento son tan extremadamente diferentes con respecto a los de los griegos que es poco provechosa la comparación". ${ }^{20}$

Estas palabras están incluidas en una nota al pie en el tomo segundo de la monumental Historia de la Filosofía Griega de W. K. C. Guthrie editada por la Universidad de Cambridge. El apartado en el que aparece esta evaluación está dedicado a Parménides y, específicamente, a "la vía falsa de las opiniones de los mortales". Allí Guthrie señala la distinción parmenídea entre el hombre al que se le ha revelado la verdad por cauces divinos y la muchedumbre de los mortales que nada saben, y expresa su sospecha de que en una primera impresión esto puede traer a la memoria del lector la noción de ilusión cósmica o maya del pensamiento hindú. Frente a tal impresión, no obstante, Guthrie remata afirmando que "la India y Parménides son dos polos opuestos". "Parménides -argumenta el autor- lleva a cabo y comprueba sus rígidas distinciones mediante un consumado razonamiento intelectual (lógos), que es para él el único suministrador de la verdad. En el brahmanismo, no sólo hay que trascender los sentidos sino también al pensamiento, al intelecto mismo, si se quiere alcanzar la verdadera realidad. La lógica es un instrumento imperfecto e inadecuado para la intuición final". ${ }^{21}$

\footnotetext{
${ }^{19}$ Para obtener un panorama orientativo a este con respecto a nivel mundial sugerimos el Rasika Report elaborado por el Dr. Andrew Nicholson y la Dra. Elisa Freschi. En él se evalúa el estado de los estudios acerca de la filosofía de la India a nivel de postgrado en las Universidades de los Estados Unidos y de Europa. Cfr. http://philosophicalrasika.com/

20 Guthrie (1969, p. 53, nota 61).

${ }^{21}$ Guthrie (1969, p. 53, nota 61).
} 
Creemos que vale la pena, en primer término, examinar algunas cuestiones de las apreciaciones que Guthrie articula siguiendo a Zimmer, una de las dos únicas referencias manifiestas que el autor brinda acerca de su conocimiento del pensamiento de la India. Podemos apreciar que el historiador distingue "las escuelas Indias" de "los griegos" pero que luego se refiere específicamente a Parménides y al brahmanismo como si estos constituyeran, en cada caso, los paradigmas del pensamiento de una y otra tradición. Nótese, asimismo, que Guthrie compara a "Parménides" con "la India" ("polos opuestos"), creyendo, tal vez, que comparar la reconstrucción de la filosofía de un pensador a partir de fragmentos, por un lado, con una tradición milenaria, por otro, es un procedimiento legítimo y en algún punto fructífero. Considérese, por otra parte, que el autor no explica qué entiende por brahmanismo ni a cuál de las diferentes tradiciones ortodoxas (pues tal categoría excluiría las numerosas tradiciones intelectuales heterodoxas indias) desarrolladas a lo largo de los siglos hace referencia. ${ }^{22}$ Cabe notar, por último, que el conocimiento que tenemos acerca de Parménides se limita a fragmentos y testimonios, con lo cual la afirmación de Guthrie acerca de la estricta y exclusiva metodología intelectual parmenídea no es más que, a lo sumo, una hipótesis inverificable. De este breve examen, en fin, de las pocas palabras con las que Guthrie sustenta su juicio relativo al pensamiento indio, podemos concluir que para un escritor y pensador con la intrepidez y la competencia suficiente para redactar una ambiciosa Historia de la Filosofía Griega, una postura tan insustancial resulta sospechosa. Si consideramos, por el contrario, que Guthrie simplemente está a tono con una opinión ampliamente difundida en su tiempo y ambiente, por lo cual su adherencia a esta no exigía mayor fundamentación, el modo en que el autor procede nos parece más comprensible.

Guthrie, en efecto, es un claro ejemplo de una posición establecida ya desde el siglo XIX al menos, que sostiene que el "pensamiento" religioso, místico y mágico de la India no puede ser calificado como filosofía. En su libro India and Europe. An essay in Philosophical Understanding, Halbfass nos brinda numerosos ejemplos de esta opinión expuesta en diferentes Historias de la Filosofía decimonónicas ${ }^{23}$ y explica en qué medida tal concepción deriva de la influencia que las traducciones latinas modernas de la doxografía antigua de Diógenes Laercio ejercieron sobre la historiografía moderna de la filosofía hasta el punto de superponerse de diversos modos con los nuevos materiales disponibles. ${ }^{24}$ Otro de los elementos que, de acuerdo con Halbfass, influyó en esta concepción es la idea, en aparente contradicción con la primera, de la universalidad de la historia y de la razón, nociones ampliamente difundidas en los siglos XVII y XVIII. La

\footnotetext{
${ }^{22}$ Compárese, por ejemplo, con las veintiún corrientes intelectuales señaladas por Tola-Dragonetti (2008, pp. 57-59). Considérese también, por ejemplo, uno de los textos más antiguos de la tradición Jaina, el Sütrakritäiga, donde se describe un panorama de las filosofías pre-budistas. En él se mencionan 363 escuelas, de las cuales 180 son kriyāvādins (creyentes en la eficacia de la acción), 84 son akriyāvādins (no creyentes en la eficacia de la acción), 67 son ajñānikas (escépticos que afirman no conocer), y 32 son vainayikas (creyentes en la salvación mediante la buena conducta). Si bien los números pueden ser exagerados revelan, de acuerdo con Mohanty, un intenso fermento intelectual. Cfr. Mohanty (1999, pp. 25-26).

23

24 Halbfass (1990, pp. 146-147).
} 
razón, no obstante, en cuyo nombre la historia de la filosofía es escrita, puede haber sido concebida efectivamente como un principio humano universal, pero el modo "adecuado" de su uso y desarrollo es algo completamente diferente. Halbfass afirma, en este sentido, que tal modulación de la concepción universalista devino el criterio con el cual juzgar un concepto de filosofía "adecuado" (proper) y "efectivo" (actual) que estaba lejos de ser universal. ${ }^{25} \mathrm{Hegel}$, agrega Halbfass, integra esta concepción tradicional en su propio pensamiento, pero la lleva a un nuevo nivel de reflexión. En su propio sistema, en efecto, el filósofo intenta preservar el pensamiento de la India y Oriental con la finalidad de alcanzar una comprensión del desarrollo histórico del concepto de filosofía, aunque tal concepto, de acuerdo con Hegel, no se encuentre necesariamente allí. $^{26}$

Ahora bien, la influencia de Hegel sobre la imagen de la India heredada en la época contemporánea merece un estudio aparte que nosotros no emprendemos aquí. Los autores F. Tola y C. Dragonetti, ya mencionados, analizan con detalle este asunto en su artículo "What Indian Philosophy owes to Hegel". ${ }^{27}$ Allí, los autores examinan la opinión ambigua de Hegel acerca de la producción intelectual india que, si bien reconoce ciertos méritos a aquella, concluye que no hay filosofía en Oriente y que no existe tal cosa como una filosofía de la India. Tola y Dragonetti demuestran, asimismo, que independientemente de los presupuestos propios del sistema hegeliano el filósofo basa sus conclusiones en un conocimiento deficiente, de segunda mano $y$, en muchos casos, errado del pensamiento indio. Esta conclusión es reforzada por las de otros autores: el ya citado Halbfass sostiene que las afirmaciones hegelianas acerca de la filosofía de la India carecen de corrección y objetividad histórica y filológica, y von Glasenapp considera que el conocimiento de Hegel acerca de la India es caricaturesco y que el pensador carecía de los requisitos necesarios para lograr la tarea que había emprendido. ${ }^{28}$ Tola y Dragonetti concluyen, en fin, que la filosofía de la India le debe a Hegel su (infundada) exclusión (de la historia de la filosofía). ${ }^{29}$

En un artículo reciente, Ankur Barua examina diversos elementos de la tradición filosófica occidental y su ascendiente sobre el dilema acerca de la existencia o no de una filosofía de la India. Así pues, el autor propone que la discusión en torno a este dilema ha tratado de definirse mediante un argumento de la siguiente forma.

Premisa 1 Philosophia es el proyecto duro (tough-minded) de investigación pura, basada en el pensamiento autónomo (theoria) y en el rechazo

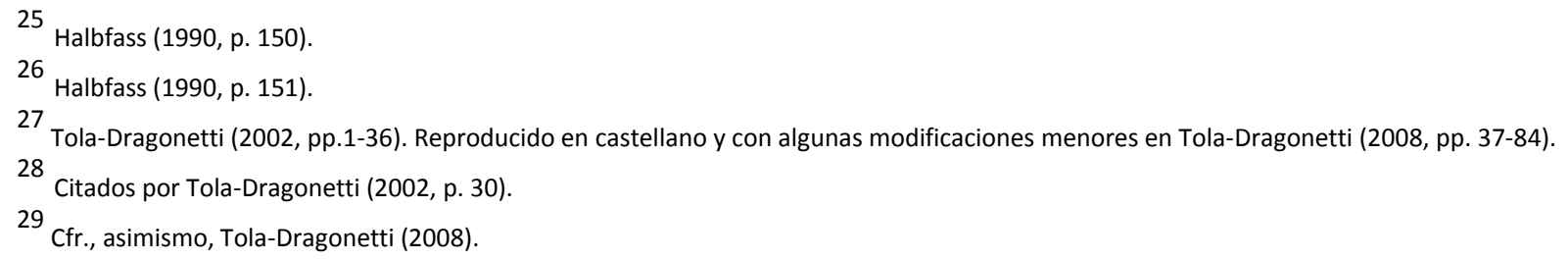


del mythos, definido como la negación del lógos, y no encadenada a ningún compromiso de fe

Premisa 2

Toda investigación en el pensamiento indio está enraizada en la especulación védica, basada en el testimonio autorizado o prácticamente orientada hacia el fin de la liberación de la existencia fenoménica. Philosophia se opone diametralmente a toda forma de pensamiento blando (tender-minded).

Conclusión Los espacios conceptuales indios están amalgamados (clustered) con la mitología, poesía, historia, dogma, teología y semejantes, pero carecen específicamente de philosophia. ${ }^{30}$

Tal argumento, señala Barua, pone de manifiesto una variedad de aspectos que requieren ser analizados. Las premisas aludidas, afirma, evidencian que la investigación acerca de este dilema es meta-filosófico, en la medida en que se indaga acerca de los límites de la empresa característicamente filosófica y de su significado en los diferentes contextos culturales. Así pues, siguiendo a Russell, el autor considera que toda definición de filosofía es controversial y que encarna una cierta actitud filosófica, además de encontrarse estructurada por fuerzas socio-históricas, culturales e institucionales. Para desarticular el argumento, no obstante, Barua ataca las dos premisas por separado. Examina, primero, diversas concepciones de philosophia en occidente, que implican, cada una, relaciones diferentes entre razón, indagación lógica y argumentación, por una parte, y fe, horizontes hermenéuticos o compromisos metafísicos subyacentes, por otra. ${ }^{31}$ Tal diversidad atentaría contra la primera premisa. Analiza, asimismo, el "trabajo de la razón" (work of reason) en el darshana indio clásico, de modo de poner en evidencia la presencia del discurso racional en la India clásica, y señala la existencia de tradiciones tanto ortodoxas como heterodoxas que no se encuentran encadenadas a la autoridad védica y que, incluso, la rechazan. Tales indicaciones contradicen la segunda premisa. ${ }^{32}$ El autor afirma, en fin, que es posible hallar numerosas clases de prácticas de la razón en las redes de tradiciones, textos y terapias propias del pensamiento clásico indio. ${ }^{33}$

Otro elemento que ha jugado un importante papel en el imaginario que opera detrás del rechazo del carácter filosófico del pensamiento de la India es su identificación con la mística. En el libro Orientalism and Religion. Postcolonial theory, India and 'the mystic East' de Richard King, el autor explora el vínculo entre la teoría poscolonial y el estudio comparativo de la religión concentrándose en la noción del "Oriente Místico". Con tal finalidad, King se propone prestar gran atención a las relaciones de poder involucradas en la historia de las ideas, examinar el modo en que la "mística", en tanto que categoría, ha sido construida en Occidente

\footnotetext{
30 Barua (2013, p. 6).

31 Barua (2013, pp. 10-15).

32 Barua (2013, pp. 16-26). En torno al concepto de autoridad en el pensamiento indio véase, asimismo, Srinivasa Rao (2007, pp. 205-220).

33 Barua (2013, p. 26).
} 
y la manera en que esta noción se ha proyectado sobre la cultura de la India con la finalidad de controlar, manipular y gestionar a Oriente. ${ }^{34}$

El autor señala, asimismo, que uno de los aspectos constitutivos de la concepción moderna acerca de la mística es su contraposición con las categorías contemporáneas de racionalidad y de filosofía, tal como estas son configuradas a partir de Kant y bajo la influencia de los estudios religiosos de William James, entre otros. ${ }^{35}$ King afirma, entonces, que tales comprensiones de la filosofía y de la mística han sido utilizadas para proyectar sobre Oriente la noción de "Oriente místico" mientras que Occidente permanecería siendo "filosófico" y racional. Estas proyecciones fallan, según King, en que procuran brindar un cuadro esencialista y homogéneo tanto de las culturas occidentales como de las asiáticas. Señala, asimismo, que asociadas con tales proyecciones se encuentran las dicotomías del Occidente como liberal, igualitario, secular y moderno, mientras que la cultura de la India sería autoritaria, jerárquica, religiosa y tradicional. Occidente ha tendido, en todos los casos, a retratarse como superior de acuerdo con sus propios criterios y la cultura de la India ha sido caracterizada como inferior. Igual de importante, agrega, ha sido la identificación de la India con lo femenino y de Occidente con lo masculino. ${ }^{36}$

En fin, son numerosos los autores que han procurado deconstruir y trazar una genealogía de la concepción según la cual el pensamiento de la India es no filosófico y no podemos agotarlos aquí ni tampoco sus argumentos. Pero tengamos en cuenta que paralelamente a tal empresa revisionista se ha desarrollado desde la segunda mitad del siglo pasado un estudio sistemático y constructivo del pensamiento de la India que, enriquecido por la crítica de los estudios postcoloniales, ha procedido con mayor conciencia y honestidad metodológica. Así pues, la opinión de los especialistas actuales en estudios hinduistas y del pensamiento indio en general es opuesta en numerosos respectos al que niega el carácter filosófico a la producción intelectual india, tal como el volumen de Tola y Dragonetti aludido al comienzo de nuestro trabajo pone de manifiesto. Este punto de vista idóneo, no obstante, todavía no es aceptado abiertamente y en la mayoría de las universidades occidentales el estudio de la filosofía de la India no está incluido en los departamentos de filosofía. En aquellas universidades que incluyen esta disciplina en su currículo, ella suele depender de los departamentos de estudios religiosos o de estudios de áreas. ${ }^{37}$ Aludamos, asimismo, a nuestro relevamiento de las treinta y dos Licenciaturas en Filosofía dictadas en nuestro país, ninguna de las

\footnotetext{
34 King (2001, p. 6).

35 El autor cita a Hart, quien afirma lo siguiente: “Lo que da vida al místico causa la muerte del filósofo: tal es la carga de la filosofía kantiana. Las distinciones fundamentales que establece la filosofía crítica -entre conocer y pensar, cognición e intuición, trabajo y gracia, la ley moral y la visión- también sirven para distinguir la filosofía de la mística. La claridad inmediata de la visión del místico debe oponerse, pues, a una claridad de otra índole que se revela en y mediante un análisis riguroso de los conceptos, una indagación lógica en el objeto (scope) y el status del conocimiento humano, una inspección de las conexiones entre las estructuras del lenguaje y del conocimiento. Por estos medios, los filósofos desde Kant han establecido una disciplina que puede mirar fuera de su fronteras con una confianza acabada, plegando a su propio territorio lo que se halla fuera, expandiéndose mediante la familiar fórmula "la filosofía de x", donde el elemento desconocido puede ser reemplazado por casi cualquier otra área de indagación." Hart (2000 [1989], pp. 210-211).

36 King (2001, pp. 31-32).

37 Lawrence (2011, pp. 140-141).
} 
cuales posee la materia Filosofía de la India, aunque tres de ellas, como hemos visto, han incluido en su más reciente plan de estudios una asignatura cuatrimestral en la que se aborda la filosofía de oriente en bloque, dentro de la cual se distingue, no obstante, el pensamiento indio del de otras tradiciones "orientales".

En las últimas décadas del siglo veinte y lo que va del nuestro, pues, se ha llevado a cabo una reevaluación crítica de la literatura de la India antigua y clásica. Se han propuesto interpretaciones renovadas y originales posibilitadas por la aplicación de técnicas filosóficas analíticas contemporáneas. Esta aproximación racional y no "mística" a la tradición intelectual india ha dado como resultado una indagación acerca de sus métodos centrales, nociones y dispositivos conceptuales. Todo esto, en fin, ha posibilitado la rehabilitación de una historia suprimida por el "orientalismo": la historia de la razón en una tierra a menudo definida como el "polo opuesto de la razón". ${ }^{38}$

Este intento de rehabilitación, no obstante, podría ser concebido como una nueva tentativa reconfigurada del "orientalismo" de buscar expresiones de la racionalidad en la India basada en los estándares impuestos por Occidente. “¿Por qué la necesidad -cuestiona Ramakrishnan- de describir la "racionalidad” de las filosofías indias, de aseverar que los filósofos de la India fueron 'tan racionales' como sus contrapartes occidentales?"39 Ganeri, sin embargo, sostiene que la empresa emprendida por él y otros tantos autores no toma la noción de "racionalidad" como un concepto occidental aplicado de mal modo e imperiosamente. Es un error, afirma, concebir la racionalidad como un concepto filosófico interno a una teoría; debe ser pensado, por el contrario, como un concepto acerca del cual hay muchas teorías. Diversas formas de racionalidad, sostiene Ganeri, se encuentran disponibles interculturalmente, aunque no siempre se hallen instanciadas interculturalmente. Desde tal perspectiva, pues, considera que es legítimo indagar acerca de la noción que la cultura india tiene sobre la racionalidad, aunque ésta se encuentre, no obstante, enmarcada, articulada y manifestada de modos culturalmente específicos. ${ }^{40}$

Chakrabarti, por su parte, en un artículo titulado "Rationality in Indian Philosophy" examina cuatro grandes preocupaciones acerca de si el concepto de racionalidad con sus connotaciones positivas es compatible con el tenor general de las filosofías clásicas de la India. ${ }^{41}$ Intenta poner de manifiesto, asimismo, que las tensiones entre testimonio escritural y razón, mística y lógica, poesía y análisis, y acción y teoría eran celebradas y discutidas por los pensadores indios antiguos y medievales. ${ }^{42} \mathrm{El}$ autor aborda, pues, los cuatro problemas relacionados de atribuir una noción de racionalidad a una tradición intelectual que suele ser

\footnotetext{
38 Ganeri (2001, p. 4 y ss.). "reason's Other" escribe el autor.

39 Ramakrishnan (1996, p. 17), citado por Ganeri (2001: 3).

40 Ganeri (2001, pp. 1-5).

41

42 Chakrabarti (1999, pp. 259-260).
} 
entendida como (1) obsesionada con aspectos prácticos y soteriológicos, (2) con una estricta adhesión a la autoridad incuestionable de los Vedas o de algún otro texto raíz, (3) que aspiraría a una penetración suprarracional, intuitiva y mística de la realidad y (4) que supuestamente carece de una noción de necesidad lógica o deductiva y de una concepción de conocimiento a priori. Chakravarti ensaya entonces diferentes respuestas a estas preocupaciones. Afirma, por una parte, la existencia de una "metaciencia" o lógica $(\bar{a} n v \bar{i} k s ̦ i k \bar{l})^{43}$ atestiguada ya en el siglo IV a. de C. en el Arthaśāstra de Kautilya, en donde se pone de manifiesto el valor teórico tanto de la indagación como de la acción racional. Señala, asimismo, las contiendas entre las vertientes filosóficas defensoras del karman (acción) y del jñāna (conocimiento), y las tensiones entre los ritualistas y los metafísicos. ${ }^{44}$ Respecto de la segunda preocupación, indica la preeminencia que en el sistema Sānikhya, a pesar de su ortodoxia, poseen la reflexión racional y sus propias concepciones acerca de los gunas y de la causalidad materialista. Señala la importancia que incluso los dos pilares de la ortodoxia védica, el Vedānta y la Mīmāmssā, otorgan al razonamiento en la argumentación crítica relacionada con la hermenéutica de los textos. Recurre también, en respuesta a este segundo problema, a concepciones occidentales contemporáneas acerca del conocimiento y a la íntima dependencia que plantean entre conocimiento y el lenguaje $y$, por ende, con la tradición. ${ }^{45}$ Respecto del supuesto misticismo imperante en la tradición india Chakrabarti señala las tradiciones anti "místicas" del Mīmāmnsā y el rechazo del Vedānta de las experiencias personales subjetivas como instancias del objetivo soteriológico trascendente que enseña. ${ }^{46} \mathrm{Y}$ en cuanto a la cuarta cuestión el autor indica el reconocimiento explícito que la lógica Nyāya-Vaiśeșika posee del principio de no contradicción, de la presencia de inferencias deductivas en la lógica budista, y del rol de los razonamientos hipotéticos como indicadores de nociones modales de necesidad entre posibilidades no actualizadas. ${ }^{47}$

En fin, luego de examinar las construcciones indias de gramáticas axiomáticas, ${ }^{48}$ la racionalidad en la ciencia médica ${ }^{49}$, la racionalidad de la concepción del dharma y de las comparaciones entre el sí mismo y el otro, y en las concepciones de la estética, Chakrabarti no conserva dudas acerca de su conclusión. ${ }^{50}$ Los diferentes sistemas filosóficos, en consonancia con la tradición pero no meramente dependiendo de ella, procuran brindar un conocimiento del sí mismo, y "este es un proyecto tan racional como lo es práctico, moral y

\footnotetext{
43 Para un examen minucioso de la noción de änvékñiké véase Halbfass (1990, cap. 15).

44 Chakrabarti (1999, pp. 262-263).

45 Chakrabarti (1999, pp. 264-267)

46 Chakrabarti (1999, pp. 267-269).

47 Chakrabarti (1999, pp. 269-272). Para un examen minucioso de la lógica india véase Matilal (1998).

48 Para un análisis de la concepción india de la gramática como ciencia véase Staal (2003, pp. 348-359).

49 Para un estudio detallado de la práctica médica científica india véase Wujastyk (2003, pp. 393-409) y (2009, pp. 67-81).

50 Chakrabarti (1999, pp. 272-277).
} 
espiritual", afirma el autor. "En lo concerniente a las más grandes corrientes filosóficas clásicas -prosigue[...] la racionalidad es estudiada teóricamente y utilizada prácticamente de modo que el agente pensante pueda perder tanto su ego individual como el yo orientado hacia los objetos exteriores y las cogniciones generadoras de deseos que vuelven al sí mismo dependiente y le quitan libertad." ${ }^{51}$ Así pues, Chakrabarti afirma que a lo largo de todo el pensamiento clásico indio pueden detectarse concepciones articuladas de lo que hace razonable a una creencia, a una acción, a una interpretación, a la elección de un medio o de un fin. Aun así, aclara que "estos conceptos tal vez no sean fácilmente reconocibles como conceptos de racionalidad dado que, a diferencia de la concepción occidental tradicional, las nociones indias típicas de racionalidad son, en promedio, no hedonísticas, no individualistas, no positivistas y apuntan a la renuncia del ego personal en virtud de una tradición impersonal o de una conciencia universal. ${ }^{52}$

Hace ya varios decenios, pues, una corriente pujante de especialistas ha venido insistiendo en la larga tradición de buen razonamiento existente en la India. Tal tradición se ha expresado a lo largo de los siglos, afirman, en diversos sistemas de gramática, epistemología, teología, metafísica, medicina, matemática, jurisprudencia, filosofía política e, incluso, estética. Frazier, por ejemplo, afirma, que el hinduismo se ha desarrollado como una cultura vigorosa de debate filosófico formal, de investigación científica y de competencia entre las escuelas de intelectuales, cultivando un enriquecido rango de disciplinas racionales que existieron en una combinación natural con la vida religiosa, las artes y la política. El estudio de la cultura intelectual india, agrega Frazier, no solo ha identificado tradiciones indias de raciocinio sino también métodos distintivos y propios de razonar, que en muchos casos van más allá de los métodos empíricos, inferenciales y verificacionistas tan comunes en el modelo analítico occidental. ${ }^{53}$ Bronkhorst, a su vez, sostiene que la India ha poseído desde la Antigüedad una tradición de indagación racional que se caracteriza por el debate racional y por el esfuerzo por parte de los pensadores de perfeccionar sus sistemas a partir de tal debate. El autor, asimismo, señala como condición de tal tradición que no existan áreas de la realidad que se excluyan del campo del examen crítico, sometidas a la autoridad exclusiva de la tradición o de la revelación. $^{54}$

Sheldon Pollock, por su parte, ha puesto de manifiesto que la tradición intelectual india se ha desplegado en el marco del auge y de la decadencia de lo que llama la "cosmópolis sánscrita". A comienzos de la era común, explica Pollock, la lengua sánscrita que durante largo tiempo había sido concebida como una lengua sagrada y restringida a la práctica religiosa fue reinventada como un código para la expresión literaria y

\footnotetext{
51 Chakrabarti (1999, p. 277).

52

Chakrabarti (1999, p. 259).

53 Frazier (2011, pp. 1-11). Considérese, asímismo, el trabajo de Staal (2014 [1988], pp. 203-229).

${ }^{54}$ Bronkhorst (1998, pp. 1-28).
} 
política. Este desarrollo marcó el comienzo de una carrera impresionante que vio a la cultura literaria sánscrita expandirse a lo largo de la mayor parte del sur de Asia. ${ }^{55}$ Este proceso se extendió hasta el comienzo del segundo milenio cuando las formas discursivas locales fueron redignificadas como lenguajes literarios y comenzaron a desafiar al sánscrito para el trabajo tanto de la literatura como de la política, finalmente reemplazándolo. ${ }^{56}$

La "cosmópolis sánscrita", pues, es la esfera trans-regional de cultura y poder del Sánscrito. ${ }^{57}$ Su desarrollo fue progresivo y tentativo, y para que pudiera surgir, explica Pollock, el entendimiento de la naturaleza y función del Sánscrito como el "lenguaje de los dioses" tuvo que transformarse. ${ }^{58}$ Una vez que el Sánscrito emergió del ambiente sacerdotal al que originalmente estaba confinado, se esparció con increíble rapidez a lo largo del Sur del Asia. En tres siglos, añade, el Sánscrito devino el único medio con el cual las elites gobernantes expresaban su poder desde el norte del actual Pakistán hasta el actual Vietnam por un lado y las planicies de Java por otro. No obstante, aclara, el Sánscrito posiblemente nunca funcionó como el lenguaje cotidiano en ninguna parte de la cosmópolis y aparentemente tampoco fue utilizado como lengua del comercio (como sí sucedió con otros códigos cosmopolitas como el Griego, el Latín, el Árabe o el Chino). El trabajo que el Sánscrito llevó a cabo estaba más allá de lo familiar y de lo instrumental, estaba dirigido, sobre todo, a articular una forma de conciencia y cultura política. Mas "política" no debe entenderse como transacciones de poder material sino como la celebración del poder estético. ${ }^{59} \mathrm{El}$ término "cosmópolis sánscrita" que acuña Pollock, pues, busca indicar los modos históricamente significativos del pasado de ser trans-local o de participar (y de saber que se está participando) en redes políticas y culturales que trascendían la comunidad inmediata. ${ }^{60}$

El autor indica, asimismo, que la introducción de los sistemas de escritura o proceso de "literalización" y el crecimiento de la cultura de los manuscritos comenzaron en la India un poco antes de la era común. A partir

\footnotetext{
55 El autor recurre para su categoría de lo literario a la concepción misma de los teóricos y practicantes de las formas dominantes de arte verbal del sur de Asia premoderna. Pollock sostiene que ellos explícitamente rechazan la idea de que el lenguaje posea una dimensión estética fuera del kavya (lo que el autor traduce como "literatura"), y derivaban de las prácticas efectivas un paradigma relativamente estable de propiedades literarias que, además de caracteres léxicos, métricos y temáticos, incluían la escritura como un componente fundamental. El autor argumenta, asimismo, que esta definición de lo literario en el Sur del Asia constituía un acto en el campo del poder. Aquellos que hacían, escuchaban y leían textos en el Sur del Asia premoderna sostenían tanto discursivamente como en la práctica una clara distinción entre literatura y no literatura. Pollock (2006, p. 5).

56 Pollock (2006, p. 1). El autor recurre a la noción de “vernacularización” para denominar este segundo proceso.

57 La frase conlleva tres implicaciones que la vuelven útil, de acuerdo con el autor: en primer lugar, su dimensión supraregional (cosmo-), que dirige la atención a la naturaleza expansiva de la formación. Segundo, es la prominencia atribuida a la dimensión política (-polis), que era de particular importancia en esa forma de identificación global. Por último, la cualificación provista por "sánscrita", que afirma el papel de este lenguaje en particular en producir las formas de expresión cultural y política a las que dio forma este orden cosmopolita. Pollock (2006, p. 12).

58

Pollock (2006, p. 12).

59 Pollock (2006, p. 14).

${ }^{60}$ Pollock describe la política imperial (imperial polity) de la cosmópolis sánscrita como territorialmente expansiva (aunque resulte complejo definir "territorio" en el marco del Sur de Asia premoderna), como políticamente universalista (aunque no sea sencillo comprender qué significaba el gobierno político) y como étnicamente no particularizada (si es que es posible utilizar el término "étnica", en tanto que no hay seguridad de que la noción político-científica de "étnica" haya existido entonces). Pollock (2006, p. 18). Afirma, asimismo, que la noción de política (rajya) y de lo literario (kavya) eran mutuamente constitutivas en tanto que cada hombre que surgió al poder buscó la distinción de la presentación de sí en la literatura sánscrita.
} 
de ese momento, la escritura, la valoración de lo escrito y la interna transformación de los textos literarios por el hecho de estar puestos por escrito devienen, de modo creciente, características prominentes de la cultura literaria. $^{61}$

En este contexto, pues, debe comprenderse el florecimiento del cultivo intelectual filosófico indio. Lawrence, en efecto, en su estudio acerca de las tradiciones filosóficas hindúes afirma que el cosmopolitanismo es un ingrediente esencial para el desarrollo de la filosofía, en el cual hay una elevada experiencia y aceptación de la diversidad cultural y donde los intelectuales están más inclinados a abordar dialógicamente los desafíos impuestos por las creencias, experiencias y prácticas alternativas. Así pues, el autor sostiene que la filosofía en la cosmópolis sánscrita fue estimulada por miles de años de encuentros entre las diversas tradiciones hindúes, y entre estas y las numerosas escuelas de budismo, jainismo, materialismo, entre otras. ${ }^{62}$

Respecto del estudio contemporáneo de la filosofía de la India, no obstante, Lawrence afirma que el investigador debe ejercitarse en la más estricta vigilancia para separar los sesgos infundados de las consideraciones intelectuales legítimas. Así pues, señala el fuerte prejuicio contra las prácticas y creencias religiosas que se encuentra profundamente arraigado en gran parte de la filosofía contemporánea occidental y el consecuente reduccionismo que las ciencias sociales han adoptado de las ciencias físicas para su explicación de los fenómenos que estudian. Menciona, asimismo, la problemática del colonialismo y del neocolonialismo articulado en los estudios académicos como "Orientalismo" en el sentido definido por Said. El prejuicio post-iluminista contra lo tradicional y el sesgo orientalista contra lo no occidental se apoyan mutuamente, según Lawrence, y han sido la causa de que grandes filósofos modernos y contemporáneos ignoraran e incluso negaran la existencia de una indagación filosófica en la India. ${ }^{63}$ La supuesta ausencia de libertad de las tradiciones indias tanto respecto de intereses prácticos y soteriológicos como de los grilletes del dogma y de la religión parece haberle privado de los requisitos de la verdadera actividad filosófica. ${ }^{64}$ Pero estos constituyen más bien prejuicios con los que los filósofos occidentales se han aproximado al estudio del pensamiento indio que conclusiones obtenidas del análisis detallado e informado de las fuentes y de las prácticas efectivas. Lawrence señala, asimismo, otros prejuicios con los que se ha abordado el pensamiento indio. Uno de ellos, y que constituye el reverso de la degradación occidental de las culturas no

\footnotetext{
${ }^{61}$ El autor señala varios sentidos en que la cultura escrita difiere de la cultura oral. Indica que la autorización para escribir y, sobre todo, para escribir literatura no es atribuida naturalmente como la capacidad de hablar, sino que se encuentra típicamente relacionada con privilegios sociales, políticos e incluso epistemológicos. La escritura, asimismo, permite por primera vez volver el discurso mismo el objeto de otro discurso. Y el lenguaje deviene, entonces, el objeto de una conciencia estetizada, el texto mismo un artefacto a ser decodificado y un pretexto para el desciframiento. Pollock (2006, p. 2).

62

63

Lawrence (2011, p. 140)

64 Halbfass (1990, p. 157).
} 
occidentales es la idealización romántica de estas como superiores en su propia alteridad. ${ }^{65}$ Otro consiste en la búsqueda de esencias universales característica de los abordajes morfológicos comparativos de la Historia de las Religiones del siglo XIX y de ocho décadas, incluso, del siglo XX. En fin, el estudio del pensamiento indio y de otras culturas, sostiene el autor, debe trascender las representaciones esencialistas y dicotómicas y debe adoptar un abordaje que aprecie los matices y variados aspectos de aquellas. ${ }^{66}$

Señalemos, en último término, que el contacto moderno entre las tradiciones ha permitido un intercambio intenso y un enriquecimiento mutuo que implicó tanto una incorporación del pensamiento filosófico occidental de parte de los estudiosos indios, como una apertura y valorización de la academia europea y americana a formas de intelectualidad ajenas y, en especial, indias. Mohanty, por ejemplo, en relación con el primero de los fenómenos mencionados comenta que hay una creciente tendencia en la India de interpretar, discutir y criticar ciertos conceptos fundamentales del pensamiento occidental en sánscrito y con el lenguaje propio de la filosofía india. ${ }^{67}$ En relación con el segundo de los fenómenos, señalemos la fundación en 1997 del Centro Oxoniense de Estudios Hinduistas, primera institución en su clase creada con la finalidad de estudiar de modo multidisciplinario la cultura hindú y de construir una interfaz entre la academia moderna y los modos indios tradicionales de estudio e investigación. Estos intercambios y enriquecimientos han propiciado un diálogo genuino entre las tradiciones filosóficas. Y si bien este diálogo debe recorrer un largo camino para establecerse firmemente, ya evidencia sus primeras germinaciones y parece prometer, del contacto y de la "reacción química" entre aquellas, una transformación mutua y la síntesis de nuevas sustancias filosóficas. Concluyamos la presente sección con unas palabras del profesor Mohanty ilustrativas en este sentido publicadas hace más de dos décadas.

¿Por qué intentar, incluso, "interpretar" la filosofía india o, para el caso, la filosofía china desde el punto de vista del pensamiento occidental? ¿No es esto una asimetría -pues la filosofía occidental no es estudiada, expuesta y criticada desde el punto de vista del pensamiento oriental-, un signo de la hegemonía cultural de occidente, de lo que Husserl llamó la "europeización de la Tierra"? Esta es, ciertamente, una pregunta muy difícil de responder... Finalmente, el objetivo debe ser sobreponernos a esta contingencia, a esta asimetría y, en lugar de interpretar una a la luz de la otra, desarrollar un discurso y una conversación en la cual los involucrados sean Platón, Bhartrhari, Aristóteles, Gautama, Vatsyayana, Dignaga, Quine, Dharmakirti, y Carnap -para mencionar solo a unos pocos. Este objetivo es lejano... pero es al menos a lo que debemos orientarnos, si la filosofía ha de ser una empresa racional. ${ }^{68}$

\footnotetext{
65 Lawrence (2011, pp. 143-144).

66 Lawrence (2011, p. 145).

67 Mohanty (1992, p. 405).

68 Mohanty (1992, p. 405).
} 


\section{Conclusiones}

A lo largo de nuestro trabajo hemos procurado discutir y dilucidar algunas cuestiones en torno a la filosofía de la India y hemos apreciado que se trata de una temática rica en complejidades que exceden ampliamente lo específicamente filosófico. En primer término, hemos examinado la situación de los estudios propios de esta disciplina en las universidades de nuestro país. El relevo de los diferentes planes de estudio de las licenciaturas en filosofía nacionales, por una parte, nos ha permitido apreciar el estado embrionario e incipiente de la disciplina en las instituciones locales. En este sentido, más que constituir una crítica, el presente análisis busca retratar la situación actual de modo de contribuir al trazado de caminos fructíferos y coherentes para el desarrollo de la disciplina a partir de un conocimiento ajustado del panorama vigente, de sus limitaciones y de sus posibilidades. En segundo término, hemos ofrecido una resumida sistematización de la polémica en torno al dilema de la (in)existencia de una filosofía de la India. Para ello, hemos reparado en algunas investigaciones de corte genealógico y deconstructivo que los especialistas han llevado a cabo con la finalidad de desarticular la concepción que sostiene que el pensamiento de la India es no filosófico. Hemos brindado, asimismo, un conciso mapa de los aspectos puestos de manifiesto por los especialistas para indicar la presencia del pensamiento filosófico en la tradición india y para resaltar el valor analítico y el carácter racional de este.

Reiteremos que este doble procedimiento ha tenido como finalidad mostrar que para poder alcanzar una comprensión adecuada de los caracteres propios de las múltiples expresiones de la intelectualidad de la India clásica, de sus posibles puntos de contacto con expresiones ajenas a la India y de sus disidencias, es necesaria la existencia de una tradición madura y autocrítica de investigación en el área específica. Tal tradición, asimismo, es indispensable para enriquecer nuestro entendimiento de la filosofía y para articular una concepción o una serie de concepciones que nos aparten de posiciones esencialistas y exclusivistas y que permitan el diálogo y posibiliten instancias de (auto)representación mediadas por el encuentro con una pluralidad de formas diversas de elaboración intelectual. Ahora bien, tal tradición específica es prácticamente inexistente en las instituciones de nuestro país dedicadas al estudio y a la enseñanza de la filosofía. Es evidente, a nuestro entender, que esta inexistencia se debe tanto a la falta de especialistas como a la herencia de concepciones acerca de la filosofía en retroceso e ignorantes del valor de la filosofía de la India que alimenta, si es posible pensarlo así, tal carencia. Consideremos, por otra parte, que la exclusión de esta área del currículo impide la posibilidad misma de justificar su ausencia dado que se suprime su problematización. Creemos, en todo caso, que la indagación en torno al pensamiento de la India 
clásica, acerca de su carácter filosófico o no filosófico, y de su consonancia o disonancia metodológica con la filosofía tal como esta es estudiada en nuestras universidades es una investigación filosófica y merece, a nuestro entender, ser tenida en cuenta a la hora de determinar el perfil de formación de los estudiantes de filosofía. Las respuestas a estos dilemas (meta) filosóficos, por lo demás, solo podrán surgir de su estudio sistemático, tal como nuestra discusión expuesta en la sección anterior intenta poner de manifiesto. Creemos, pues, que el único camino por delante para las licenciaturas en filosofía de nuestro país en cuanto al tema que hemos estado analizando parecería ser el de una progresiva incorporación de la filosofía de la India en su currículo y el de una creciente búsqueda por implementar instancias de formación de especialistas que puedan llevar adelante y sostener esta inclusión. Tres universidades han dado un primer paso, las restantes las irán siguiendo. Tal pronóstico es, a nuestro entender, favorable.

\section{Bibliografía Citada}

BARUA, A. (2013). "Is there 'philosophy' in India? An Exercise in Meta-philosophy." En Studies in Humanities and Social Sciences, Vol. 20, No. 1.

BRONKHORST, J. (1998). "Why is there Philosophy in India?". Sixth Gonda Lecture, held on 13 November on the premises of the Royal Netherlands Academy of Arts and Sciences. Amsterdam.

CHAKRABARTI, A. (1999). "Rationality in Indian Philosophy". En DEUTSCH, E. and BONTEKOE, R. (eds.). A Companion to World Philosophies. Oxford: Blackwell, pp 259-278.

FLOOD, G. (ed.). (2003). The Blackwell Companion to Hinduism. Oxford: Blackwell Publishing, FRAZIER, J. (2011). "Reason and Rationality in Hindu Studies". En The Journal of Hindu Studies, vol. 4, pp. 111.

FRESCHI, E. (2014). PhD programs in Indian Philosophy. Part 2: Europe. Posted on 26 March 2014. http://indianphilosophyblog.org/2014/03/26/phd-programs-in-indian-philosophy-part-2-europe/

GANERI, J. (2001). Philosophy in Classical India. The proper work of reason. London, Routledge.

GUTHRIE, W. K. (1969). A History of Greek Philosophy. Volume II. The presocratic tradicion from Parmenides to Democritus. Cambridge: Cambridge University Press.

HALBFASS, W. (1990). India and Europe: an essay in philosophical understanding. Albany: SUNY Press.

HART, K. (1989). The Trespass of the Sign: Deconstruction, Theology and Philosophy. Cambridge: Cambridge University Press.

KING, R. (1999). Orientalism and Religion. Postcolonial theory, India and 'the mystic East'. London: Routledge.

KRISHNA, D. (1991). Indian Philosophy. A counter perspective. Delhi: Oxford University Press. 
LAWRENCE, D. P. (2011). "Hindu Philosophical Traditions". En FRAZIER, J. (ed.). The Continuum Companion to Hindu Studies. London \& New York: Continuum International Publishing Group, pp. 137-151.

MATILAL, B. K. (au.), TIWARI, H. y GANERI, J. (eds.). (1998). The Character of Logic in India. New York: SUNY Press.

MOHANTY, J. N. (1999). "A History of Indian Philosophy". En DEUTSCH, E. And BONTEKOE, R. (eds.). A Companion to World Philosophies. Oxford: Blackwell, pp 24-48.

NICHOLSON, A. (2014). The Philosophical Rasika Report: Listings of Ph.D. Programs in Indian Philosophy. Part I: North America: Posted on 17 March 2014. http://indianphilosophyblog.org/2014/03/17/thephilosophical-rasika-report-listings-of-ph-d-programs-in-indian-philosophy-part-i-north-america/

POLLOCK, Sh. (2006). The Language of the Gods in the World of Men.Sanskrit, Culture, and Power in Premodern India. Berkeley and Los Angeles, University of California Press.

RAM-PRASAD, C. (2003). “Contemporary Political Hinduism”. En FLOOD, G. (ed.). The Blackwell Companion to Hinduism. Oxford, Blackwell Publishing, pp. 527-550.

SRINIVASA RAO (2007). "Subordinate' or 'Supreme'? The Nature of Reason in India and the West". En Beyond Orientalism. The Work of Wilhelm Halbfass and its Impact on Indian and Cross-Cultural Studies. Delhi: Motilal Banarsidass Publishers Private Limited, pp. 205-220.

STAAL, F. (2003). "The Indian Science of Language". En FLOOD, G. (ed.). The Blackwell Companion to Hinduism. Oxford: Blackwell Publishing, pp. 348-359.

STAAL, F. (2014). "Is there Philosophy in Asia". En LARSON, G, y DEUTSCH, Eliot. Interpreting Across Boundaries: New Essays in Comparative Philosophy. New Jersey: Princeton University Press, Pp. 203-229.

TOLA, F. y DRAGONETTI, C. (2002). "What Indian Philosophy owes to Hegel”. En Annals of the Bhandarkar Oriental Research Institute, vol. LXXXIII, pp. 1-36.

TOLA, F. Y DRAGONETTI, C. (2008). Filosofía de la India. Del Veda al Vedanta. El sistema Samkhya. El mito de la oposición entre "pensamiento" indio y "filosofía" occidental. Barcelona: Kairós.

WUJASTYK, D. (2003). “The Science of Medicine”. En FLOOD, G. (ed.). The Blackwell Companion to Hinduism. Oxford: Blackwell Publishing, pp. 393-409.

WUJASTYK, D. (2009). “Post-Classical Indian Traditions of Medical Debate and Argumentation. The Rogārogavāda of Vïreśvara". En eJournal of Indian Medicine 3, pp. 67-81. 